\title{
A Molecular Hydrogen Production Model from Li and LiH in the Early Universe
}

\author{
Raúl G. E. Morales*, Mauricio R. Canales \\ Centre for Environmental Sciences, Faculty of Sciences, University of Chile, Santiago, Chile \\ Email: *correo@raulmorales.cl
}

Received February 14, 2013; revised March 15, 2013; accepted March 22, 2013

Copyright (C) 2013 Raúl G. E. Morales, Mauricio R. Canales. This is an open access article distributed under the Creative Commons Attribution License, which permits unrestricted use, distribution, and reproduction in any medium, provided the original work is properly cited.

\begin{abstract}
Neutral isotopes and ions of $\mathrm{H}, \mathrm{He}$ and Li define the chemistry of the early universe by collisional and radiative process, where under low temperature and radiation regime, only neutral species were essential in the cooling mass that gave origin to the first proto star structures. Nevertheless, up to now, in every kinetic model $\mathrm{Li}$ is permanently discarded from fundamental reactions due to its extremely low density. Contrarily to these previous models we have developed a novel kinetic model based on two consecutive reactions of $\mathrm{Li}$ and $\mathrm{LiH}$ with $\mathrm{H}$, in order to generate a recursive process that fit well $\mathrm{H}_{2}$ production to temperatures as low as $200 \mathrm{~K}$, according to the cosmological time at the end of the dark epoch. Our results show how $\mathrm{Li}$ and $\mathrm{LiH}$ merge as first catalyzers of the $\mathrm{H}$ to $\mathrm{H}_{2}$ chemical reaction and permit us to explain the expected abundance of $\mathrm{H}_{2}$ as the main coolant in the early universe as well as in cold regions of the cosmos.
\end{abstract}

Keywords: $\mathrm{H}_{2} ; \mathrm{Li}$ and $\mathrm{LiH}$ in the Dark Epoch; $\mathrm{Li}$ and $\mathrm{LiH}$ as Primitive Catalyzers; $\mathrm{H}_{2}$ Production from Li Model

\section{Introduction}

The standard Big Bang theory permits to explain how the universe had been expanding adiabatically, cooling from an initial high extreme condition of temperature and density [1]. After the first second neutrons and protons initiate the fusion reactions that set the beginning of nucleosynthesis stage at temperatures of $10^{10} \mathrm{~K}$, where 100 seconds later this process ceases being formed mainly $\mathrm{H}$ and $\mathrm{He}$, as well as in minor quantities Li and several cations and anions derived from these initial nuclear species under the denomination of the recombination epoch. Thus, after that free electrons take part in the atoms and ions materialization, the universe begins a new phase known as the thermal decoupling of matter and radiation while expansion and cooling continue, and the $\mathrm{H}_{2}$ molecule became one of the most fundamental coolant in the low temperature range [2].

The chemistry of the early universe is broad and diverse, whenever only few atomic species were the main reactants: $\mathrm{H}, \mathrm{He}, \mathrm{Li}$ and the isotopic forms and ions. Furthermore, the chemistry of these atomic and molecular reactants and products were mainly governed by both collisional and radiative process in gas state [3]. Some

${ }^{*}$ Corresponding author. authors [4-6] have intended to represent the first proto star formations at this stage of the early universe, denominated dark epoch, by chemical reaction networks based on the neutral primordial molecules such as $\mathrm{H}_{2}$ and $\mathrm{HD}$ as well as $\mathrm{H}, \mathrm{D}, \mathrm{He}$ and their corresponding atomic cations and anions. However, due to the high relative abundance of $\mathrm{H}$ respect to the remainder atoms, $\mathrm{H}_{2}$ emerges in the universe as a primordial molecule due its high abundance and maintains a high interest in all the constitutive models of the first proto stars.

Nevertheless, in spite of the different theoretical approaches respect to generate $\mathrm{H}_{2}$, the particular role of the primordial $\mathrm{Li}$ as a precursor of this molecular species has not been suitably visualized up to date, particularly, in consideration to the low abundance calculated for the early universe. Effectively, Stancil et al. [7] determined the chemistry of primordial gas $\mathrm{LiH}$ in protoclouds through a broad set of fundamental reactions suggesting a primitive $\mathrm{LiH}$ abundance of $10^{-18}$ respects to the atomic hydrogen abundance as the unity. In general, this kind of primordial molecules with dipole moment has been mostly explored in order to analyze the specific role as cooling centers for radiative process immerse in atomic hydrogen big clouds, but the singular case of $\mathrm{LiH}$, in different works, has been permanently discarded as an suitable species for chemical reactions due to its ex- 
tremely low abundance [8].

In the early universe, $\mathrm{H}_{2}$ has been mainly conceived as product of radiative process, derived from $\mathrm{H}^{-}, \mathrm{H}^{+}, \mathrm{H}_{2}{ }^{+}$ and $\mathrm{H}_{3}{ }^{+}$reactions [3,9]. Contrarily to these current models, we have repaired in a new role of $\mathrm{Li}$ and $\mathrm{LiH}$ species as precursors of $\mathrm{H}_{2}$, in two concerted and consecutive reactions due to Li can act as a catalyzer for neutral reactions, particularly at low densities and temperatures. Thus, the $\mathrm{Li}+\mathrm{H}$ and $\mathrm{LiH}+\mathrm{H}$ reactions are an effective and alternative route to the ionization processes and the same time permit us to understand in a best way the role of hydrogen at the end of the dark epoch, previous to the first proto star formations under low radiation and temperature regime [10].

Therefore, in the present work, we have introduced a chemical model that considers the $\mathrm{H}_{2}$ production from neutral atom reactions between $\mathrm{Li}$ and $\mathrm{H}$, and the subsequent reaction between $\mathrm{LiH}$ and $\mathrm{H}$, both cases from a collisional-radiative kinetic mechanism, under physicochemical conditions of variable low density and temperature ranges characteristics of expected primitive hydrogen cold nebulas or first generation of proto-stellar formation. Our hypothesis shows a recursive behavior of lithium reactions, which determine the onset of molecular hydrogen, even though these lithium species are found in very low abundance relative to atomic hydrogen density, in a similar behavior to catalyzer reactant.

\section{Materials and Methodology}

The kinetic model applied to the molecular hydrogen production comprise a collisional process scheme of two consecutive reactions between $\mathrm{H}$ and $\mathrm{Li}$ atoms in the first event and between $\mathrm{H}$ and $\mathrm{LiH}$ in the second one, all these species prevailing in the same bulk of cold gases, with temperatures lower than $200 \mathrm{~K}$, such as must be expected at the end of the dark epoch in the early universe. Furthermore, according to the last calculations developed by Larena et al. [11] about $\mathrm{H}$ and $\mathrm{Li}$ in the primitive universe, we have used their density relationship of $\mathrm{Li} / \mathrm{H}=$ $2.387 \times 10^{-10}$. Therefore, we have assumed essentially a classical collisional-radiative kinetic model for $\mathrm{Li}$ and $\mathrm{H}$ in the first reaction and a classic collisional approach for $\mathrm{LiH}$ and $\mathrm{H}$, in the second one, as two consecutive reactions governed by a kinetic constants $k_{0}$ and $k_{1}$, respectively, in a typical scheme of pseudo-first order reactions, where the relative abundance determine that $\mathrm{H} \gg \mathrm{Li}$ and $\mathrm{H} \gg \mathrm{LiH}$.

Thus, the following kinetic scheme can be described for the two main reactions and their corresponding equations in a pseudo-first order approach as:

$$
\mathrm{Li}(2 \mathrm{~s})+\mathrm{H}(1 \mathrm{~s}) \rightarrow \mathrm{LiH}\left(\mathrm{X},{ }^{1} \Sigma^{+}\right)
$$

and

$$
\begin{gathered}
\{\mathrm{d}[\mathrm{LiH}] / \mathrm{d} t\}=k_{0}[\mathrm{Li}] \\
\mathrm{LiH}\left(\mathrm{X},{ }^{1} \Sigma^{+}\right)+\mathrm{H}(1 \mathrm{~s}) \rightarrow \mathrm{Li}(2 \mathrm{~s})+\mathrm{H}_{2}\left(\mathrm{X},{ }^{1} \Sigma^{+}\right)
\end{gathered}
$$

and

$$
\left\{\mathrm{d}\left[\mathrm{H}_{2}\right] / \mathrm{d} t\right\}=k_{1}[\mathrm{LiH}]
$$

where $k_{0}=k_{0}^{\prime}[\mathrm{H}]$ and $k_{1}=k_{1}^{\prime}[\mathrm{H}]$, and the final concentrations of $[\mathrm{Li}],[\mathrm{LiH}]$ and $\left[\mathrm{H}_{2}\right]$ are determined according to the following equations $[12,13]$ :

$$
\begin{gathered}
{[\mathrm{Li}(t)]=[\mathrm{Li}]_{0} \mathrm{e}^{-k_{0} t}} \\
{[\mathrm{LiH}(t)]=[\mathrm{Li}]_{0}\left[k_{0} /\left(k_{1}-k_{0}\right)\right]\left(\mathrm{e}^{-k_{0} t}-\mathrm{e}^{-k_{1} t}\right)} \\
{\left[\mathrm{H}_{2}(t)\right]=[\mathrm{Li}]_{0}\left(1-\left[k_{0} /\left(k_{1}-k_{0}\right)\right] \mathrm{e}^{-k_{0} t}\right.} \\
\left.+\left[k_{1} /\left(k_{1}-k_{0}\right)\right] \mathrm{e}^{-k_{1} t}\right)
\end{gathered}
$$

From a computational point of view the integration of the kinetic equations were done analytically according to Equations (3) to (5), which means that the abundance of species depend on the time evolution directly and no time steps are used as in case of numerical integration [14]. Consequently, the abundances of $\left[\mathrm{H}_{2}\right]$ as the final product depend on the initial density of $[\mathrm{Li}]_{0}$ and the inner kinetic constants ( $k_{0}^{\prime}$ and $\left.k_{1}^{\prime}\right)$. The computational procedure registers the abundances of $[\mathrm{Li}],[\mathrm{LiH}]$ and $\left[\mathrm{H}_{2}\right]$, where in this last species we have differenced two situations, by stepwise as $\left[\mathrm{H}_{2}(t)\right]$ and by accretion as $\left[\mathrm{H}_{2}\right]_{\mathrm{T}}$.

This kinetic model was implemented in IDL 6.1 software and once established the initial code we searched its operating limits from $2.4 \times 10^{4}$ to 2.4 (atoms $\mathrm{cm}^{-3}$ ) for $[\mathrm{H}]_{0}$ and from $1.0 \times 10^{-6}$ to $1.0 \times 10^{-10}\left(\right.$ atoms cm ${ }^{-3}$ ) for $[\mathrm{Li}]_{0}$. The calculations were implemented in a Power Edge T710 computer with 18 cores Intel Xeon X5670 and double precision data type of 64 bite and $48 \mathrm{~GB}$ of memory.

\section{Results and Discussion}

For hydrogen high density conditions, beyond $1.0 \times 10^{4}$ (atoms $\mathrm{cm}^{-3}$ ) and $200 \mathrm{~K}$ temperature, a three body collisional approach determines the normal course of the classical gaseous chemical process, where the reaction rate coefficients of the equations 1 and 2 are $k_{0}^{\prime}=4.64 \times$ $10^{-10} \mathrm{~cm}^{3} \cdot \mathrm{s}^{-1}$ and $k_{1}^{\prime}=1.87 \times 10^{-10} \mathrm{~cm}^{3} \cdot \mathrm{s}^{-1}$ for the first and the second reactions [15], respectively. In this case, the $\left(k_{1}^{\prime} / k_{0}^{\prime}\right)$ ratio reaches 0.403 and, therefore, the system follows a classical kinetic approach for a consecutive two steps reaction $[12,13]$. This kind of chemical reactions can typically be expected when the system moves to the Jeans' condition, previous to the final step of each primitive proto-star hydrogen cold nebula formation. In Figure 1, we show the classical chemical dynamic of Li, $\mathrm{LiH}$ and $\mathrm{H}_{2}$, for a singular case of hydrogen atomic den- 
sity nebula of $10^{4}$ (atoms $\mathrm{cm}^{-3}$ ) according to our model.

In Figure 1 the maximum of $\mathrm{Li}$ consumption coincides with the maximum of $\mathrm{LiH}$ formation and the $\mathrm{H}_{2}$ product obtained from every reaction step describes a sigmoidal curve up to reach the Li recuperation. However the total $\mathrm{H}_{2}$ obtained by accumulation of every cycle, due to the catalyst effect of the $\mathrm{LiH}$ after equation 2, begin an exponential progression. According to this approach, the total molecular hydrogen density formation reaches $1.0 \times 10^{-5}$ (molecules $\mathrm{cm}^{-3}$ ) after three centuries. This typical density can be observed in different studies as a critical value of $\mathrm{H}_{2}$ in order to reach a significant role as molecular coolant at low temperatures near to 200 $\mathrm{K}$ [4].

However, in order to analyze tenuous and cold atomic hydrogen nebulas, the three body classical kinetic approach could not be used due to the low collisional probabilities of the reactants. Thus, the chemical reactions only can occur after spontaneous radiative association. Therefore, we have made use of the spontaneous radiative rate coefficient for the reaction: $\mathrm{Li}(2 \mathrm{~s})+\mathrm{H}(1 \mathrm{~s})$ determined by Bennet et al. [16], which reaches $3.75 \times$ $10^{-20}\left(\mathrm{~cm}^{3} \cdot \mathrm{s}^{-1}\right)$; while for the second reaction rate coefficient between $\mathrm{LiH}\left(\mathrm{X},{ }^{1} \Sigma^{+}\right)$and $\mathrm{H}(1 \mathrm{~s})$ we have made use of the quasi-classical trajectories reaction determined by Dunne et al. [17], which reaches a rate coefficient of $k_{1}^{\prime}=1.92 \times 10^{-10}\left(\mathrm{~cm}^{3} \cdot \mathrm{s}^{-1}\right)$, both constants calculated at $200 \mathrm{~K}$. This situation modifies strongly the current conditions, due to the $\left(k_{1}^{\prime} / k_{0}^{\prime}\right)$ rate coefficient change from 0.403 to $5.12 \times 10^{9}$.

In Figure 2 we show the expected kinetic behavior for these two consecutive reactions ( 1 and 2 ) with a sponta- neous radiative association between $\mathrm{H}$ and $\mathrm{Li}$ as a singular case which can be extrapolated to low atomic hydrogen density nebulas of 1 (atom $\mathrm{cm}^{-3}$ ) where Li can reach typical densities of $10^{-10}$ (atoms $\mathrm{cm}^{-3}$ ). The extremely low and slow variations of $\mathrm{LiH}$ under these new kinetics conditions determine only small changes in Li abundance where after five centuries reaches a constant value, following a typical catalyzer behavior. After that point, $\mathrm{H}_{2}$ by steps follows up to reach the Li density, while the total $\mathrm{H}_{2}$ by accumulation growth in an exponential progression. Finally, this last species reaches a density of 1.0 $\times 10^{-5}$ (molecules $\mathrm{cm}^{3}$ ) only after 400 million years, which compare very well to the time ranges that determine the birth lifetime of primitive stars.

Stancil et al. [7] have rigorously discussed the Lithium Chemistry in the post-recombination epoch in order to reach a more comprehensive known of this period. However, the $\mathrm{LiH}$ abundance has been qualified as insignificant because the low rate coefficient for the radiative association between lithium and hydrogen [7]. This result determined that $\mathrm{LiH}$ was disesteemed with an insignificant role in erasing primary anisotropies in the cosmic background radiation in spite of its particular big dipolar moment [4]. On the other hand, $\mathrm{LiH}$ as potential molecular cooling on a primordial element of gas, was discarded too, due to this species would need an abundance at least ten orders of magnitude higher to be an efficient cooler, so $\mathrm{LiH}$ was ruled out as an important cooler in primordial gas $[8,18,19]$.

Under these considerations $\mathrm{LiH}$ was understood as negligible molecular system in the early universe and therefore unable to induce noteworthy changes in the star evolution. Therefore, from different analysis of the chemical

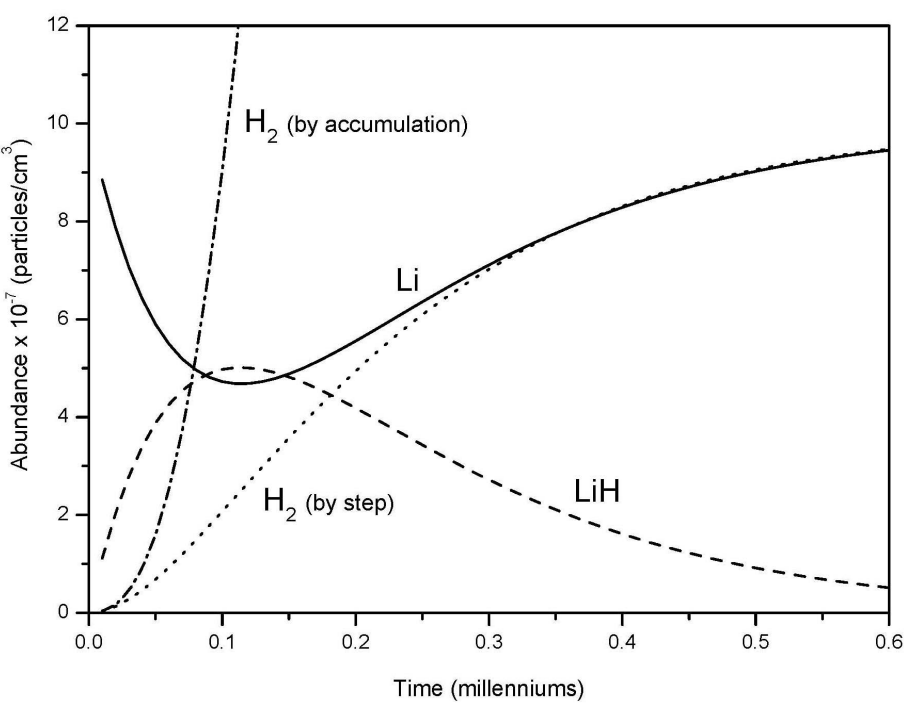

Figure 1. Molecular productions of $\mathrm{H}_{2}$ and $\mathrm{LiH}$ from chemical reactions derived from the present model due to classical collisional association between $\mathrm{Li}+\mathrm{H}$, as well as $\mathrm{LiH}+\mathrm{H}$, for high atomic hydrogen density nebula and $\mathrm{Li}$ density of $1.0 \times 10^{-6}$ $\left(\right.$ atoms $\mathrm{cm}^{-3}$ ) at $200 \mathrm{~K}$. 


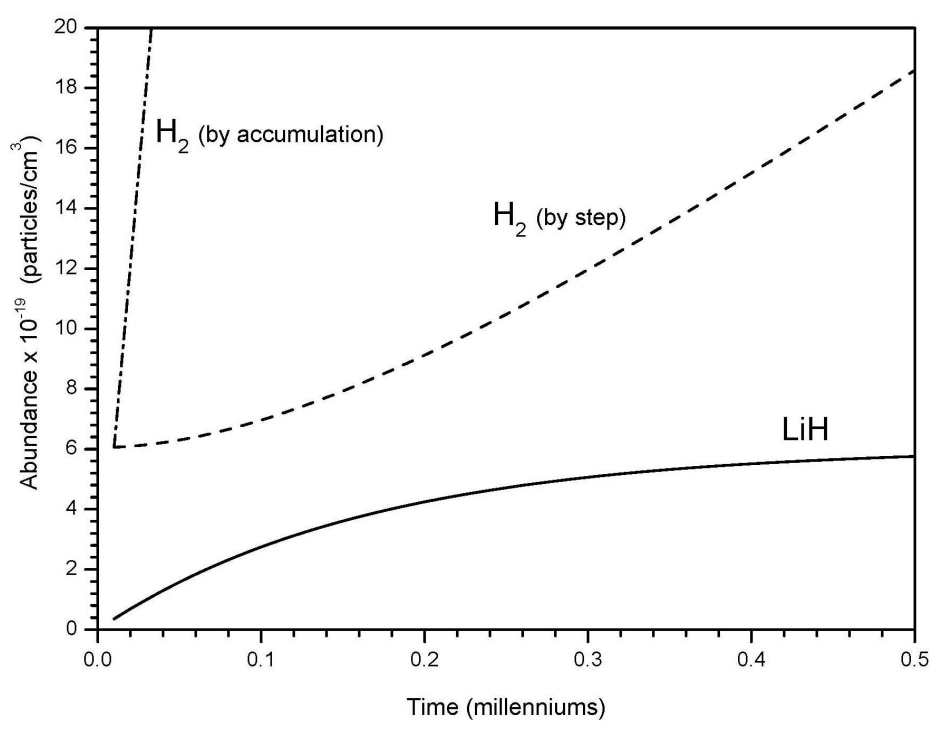

Figure 2. Molecular productions of $\mathrm{H}_{2}$ and $\mathrm{LiH}$ from chemical reactions derived from the present model due to collisional and spontaneous radiative association between $\mathrm{Li}+\mathrm{H}$, as well as $\mathrm{LiH}+\mathrm{H}$, for low atomic hydrogen density nebula and $\mathrm{Li}$ density of $1.0 \times 10^{-10}\left(\right.$ atoms $\left.\mathrm{cm}^{-3}\right)$ at $200 \mathrm{~K}$.

reactions in the early universe, $\mathrm{Li}$ has not been suitably included in reaction networks of $\mathrm{H}_{2}$ formation [20]. However, the catalyzer role of $\mathrm{Li}$ emerges as an interesting and new aspect to be considered in the prestellar molecular hydrogen under low temperature and photoionization regime, as well as in the evolution of proto-star hydrogen nebulae. As can be seen, our model follows well the expected trends of $\mathrm{H}_{2}$ formation under $200 \mathrm{~K}$, where the species are projected to be mainly in ground state due to the low UV radiation. By following, the well-known ionization reactions of $\mathrm{H}^{-}, \mathrm{H}^{+}, \mathrm{H}_{2}^{+}$and $\mathrm{H}_{3}^{+}$for molecular hydrogen production could not efficiently apply due to only fluxes greater than $5 \times 10^{-15} \mathrm{erg} \mathrm{cm}{ }^{-2} \cdot \mathrm{s}^{-1} \cdot \mathrm{Hz}^{-1} \cdot \mathrm{sr}^{-1}$ converts UV pumping effective in order to increase populations of excited molecules [20]. Thus, all these atomic species are predominantly expected to be in ground state, and the $\mathrm{H}_{2}$ production, contrarily to other hypothesis, can be simply explained by means of our $\mathrm{Li} / \mathrm{LiH}$ catalyzer model [21].

Finally, our model based in consecutive reactions of $\mathrm{Li}$ and $\mathrm{LiH}$, for the molecular hydrogen production at low temperatures and radiation regime permits us to reinforce the coolant effect of $\mathrm{H}_{2}$ under $200 \mathrm{~K}$. In addition, our model involving these two Li consecutive pseudo-first order reactions at low levels of $\mathrm{H}$ density could satisfactory explain the yield of $\mathrm{H}_{2}$ in early steps previous to the $\mathrm{H}$ gravity collapse during the first star formation, in which so far only ions-radiative processes involving $\mathrm{H}$ has been postulated up to date $[20,22]$. Furthermore, our model can be extended to other cosmic cold $\mathrm{H}$-environment where the presence of high densities levels of $\mathrm{H}_{2}$ represents a challenge to our known.

\section{Conclusion}

This work establishes for the first time a new role for $\mathrm{Li}$ and $\mathrm{LiH}$ as two main catalyzer species of the $\mathrm{H}_{2}$ production in the early universe. Furthermore, the $\mathrm{H}_{2}$ molecular production at low temperatures and radiation regime can be well explained by means of two chemical reaction of neutral species, where both consecutive reactions of $\mathrm{Li}$ and $\mathrm{LiH}$ with $\mathrm{H}$, determine a continue production of $\mathrm{H}_{2}$ reaching observed densities in good agreement to typical scale times for proto star evolution as well as cold regions of the cosmos.

\section{Acknowledgements}

The authors' acknowledgement to the Centre for Environmental Sciences of the University of Chile for financial support.

\section{REFERENCES}

[1] P. J. E. Peebles, "Principles of Physical Cosmology," Princeton University Press, Princeton, 1993.

[2] S. Weinberg, "The First Three Minutes: A Modern View of the Origin of the Universe," Published by Basic Books, Perseus Books Group, New York, 1993.

[3] A. Dalgarno, "The Growth of Molecular Complexity in the Universe," Faraday Discussions, Vol. 133, 2006, pp. 925. doi:10.1039/b605715b

[4] D. Galli and F. Palla, "The Chemistry of the Early Universe," Astronomy \& Astrophysics, Vol. 335, 1998, pp. 403-423.

[5] S. Bobino, M. Tacconi, F. A. Gianturco, D. Galli and F. Palla, "On the Relative Abundance of $\mathrm{LiH}$ and $\mathrm{LiH}^{+} \mathrm{Mo}-$ lecules in the Early Universe: New Results from Quantum 
Reactions,” Astrophysical Journal, Vol. 731, 2011, p. 107. doi:10.1088/0004-637X/731/2/107

[6] M. Tegmark, J. Silk, M. J. Rees, A. Blanchard, T. Abel and F. Palla, "How Small Were the First Cosmological Objects?" Astrophysical Journal, Vol. 474, No. 1, 1997, pp. 1-12. doi: $10.1086 / 303434$

[7] P. C. Stancil, S. Lepp and A. Dalgarno, "The Lithium Chemistry of the Early Universe," Astrophysical Journal, Vol. 458, No. 401, 1996, pp. 401-406. doi:10.1086/176824

[8] P. C. Stancil and A. Dalgarno, "Stimulated Radiative Association of Li and H in the Early Universe," Astrophysical Journal, Vol. 479, No. 2, 1997, pp. 543-546. doi: $10.1086 / 303920$

[9] T. de Jong, "The Density of $\mathrm{H}_{2}$ Molecules in Dark Interstellar Clouds," Astronomy \& Astrophysics, Vol. 20, 1972, pp. 263-274.

[10] E. Bodo, F. A. Gianturco and R. Martinazzo, "The GasPhase Lithium Chemistry in the Early Universe: Elementary Processes, Interaction Forces and Quantum Dynamics," Physics Reports, Vol. 384, No. 3, 2003, pp. 85-119. doi:10.1016/S0370-1573(03)00243-6

[11] J. Larena, J. M. Alimi and A. Serna, "Big Bang Nucleosynthesis in Scalar Tensor Gravity: The Key Problem of the Primordial ${ }^{7} \mathrm{Li}$ Abundance," Astrophysical Journal, Vol. 658, No. 1, 2007, pp. 1-10. doi:10.1086/511028

[12] I. Levine, "Physical Chemistry," 5th Edition, McGraw Hill, New York, 2005.

[13] P. Atkins and J. De Paula, "Physical Chemistry," 8th Edition, Oxford University Press, New York, 2006.

[14] H. Margenau and G. M. Murphy, "The Mathematics of Physics and Chemistry," Editorial Van Nostrand, 1956.

[15] S. Lepp, P. C. Stancil and A. Dalgarno, "Atomic and Molecular Processes in the Early Universe," Journal of Phy- sics B: Atomic, Molecular and Optical Physics, Vol. 35, No. 10, 2002, pp. R1-R24.

doi:10.1088/0953-4075/35/10/201

[16] O. J. Bennet, A. S. Dickinson, T. Leininger and F. X. Gadea, "Radiative Association in $\mathrm{Li}+\mathrm{H}$ Revisited: The Role of Quasi-Bound States," Monthly Notices of the Royal Astronomical Society, Vol. 341, No. 1, 2003, pp. 361-368. doi:10.1046/j.1365-8711.2003.06422.x

[17] L. J. Dunne, J. N. Murrell and P. Jemmer, "Analytical Potential Energy Surface and Quasi-Classical Dynamics for the Reaction $\mathrm{LiH}\left(\mathrm{X},{ }^{1} \Sigma+\right)+\mathrm{H}\left({ }^{2} \mathrm{~S}\right)>\mathrm{Li}\left({ }^{2} \mathrm{~S}\right)+\mathrm{H}_{2}\left(\mathrm{X},{ }^{1} \Sigma \mathrm{g}+\right)$," Chemical Physics Letters, Vol. 336, No. 1-2, 2001, pp. 1-6. doi:10.1016/S0009-2614(01)00102-6

[18] H. Mizusawa, K. Omukai and R. Nishi, "Primordial Molecular Emission in Population III Galaxies," Publication of the Astronomy Society of Japan, Vol. 57, 2005, pp. 951-967.

[19] J. P. Prieto, P. Padoan, R. Jimenez and L. Infante, "Population III Stars from Turbulent Fragmentation at Redshift 11," Astrophysics Journal, Vol. 731, 2011, p. L38. doi:10.1088/2041-8205/731/2/L38

[20] T. Abel, P. Anninos, Y. Zhang and M. L. Norman, "Modeling Primordial Gas in Numerical Cosmology," New Astronomy, Vol. 2, No. 3, 1997, pp. 181-207. doi:10.1016/S1384-1076(97)00010-9

[21] M. Canales and R. G. E. Morales, "A Kinetic Model for $\mathrm{H}_{2}$ Formation from Li in Pre-stellar Atmospheres," Proceedings of the 7th Congress of Chilean Environmental Chemistry and Physics-Atmospheric Section, Concepción, 20-22 October 2011, p. 2.

[22] V. Bromm, P. S. Coppi and R. B. Larson, "The Formation of the First Stars. I. The Primordial Star-forming Cloud," Astrophysical Journal, Vol. 564, No. 1, 2002, pp. 23-51. doi:10.1086/323947 